\title{
Cannabis and psychosis: the impact of polydrug use
}

\author{
Mark Shevlin, Eoin McElroy, Jamie Murphy, Philip Hyland, Frédérique Vallieres, Ask Elklit and \\ Mogens Christoffersen
}

\author{
Mark Shevlin is a Professor at \\ the Psychology Research \\ Institute, School of Psychology, \\ Ulster University, Derry, UK. \\ Eoin McElroy and Jamie Murphy \\ are both based at the \\ Psychology Research Institute, \\ School of Psychology, Ulster \\ University, Derry, UK. \\ Philip Hyland is based at the \\ National College of Ireland, \\ Dublin, Ireland and School of \\ Psychology, Centre for Global \\ Health, Trinity College Dublin, \\ Ireland. \\ Frédérique Vallieres is based at \\ the School of Psychology, \\ Centre for Global Health, Trinity \\ College Dublin, Ireland. \\ Ask Elklit is based at the National \\ Centre for Psychotraumatology, \\ Institute for Psychology, \\ University of Southern Denmark, \\ Odense, Denmark. \\ Mogens Christoffersen is \\ based at the Danish National \\ Centre for Social Research, \\ Copenhagen, Denmark.
}

Received 31 March 2017 Revised 9 May 2017 Accepted 2 June 2017

The authors assert that all procedures contributing to this work comply with the ethical standards of the relevant national and institutional committee on human experimentation with the Helsinki Declaration of 1975, as revised in 2008. The authors assert that ethical approval for publication of this audit has been provided by their local REC. This research received no specific grant from any funding agency, commercial or not-for-profit sectors.

\begin{abstract}
Purpose - While research has consistently identified an association between cannabis use and psychosis, few studies have examined this relationship in a polydrug context (i.e. combining cannabis with other illicit substances). The paper aims to discuss this issue.

Design/methodology/approach - The present study sought to examine the association between recreational drug use (cannabis only vs polydrug) and psychotic disorders. Analysis was conducted on a large, representative survey of young Danish people aged $24(n=4,718)$. Participants completed self-report measures of lifetime drug use and this information was linked to the Danish psychiatric registry system.

Findings - Multivariate binary logistic regression analysis was used to examine the association between drug use (no drug use, cannabis only, cannabis and other drug) and ICD-10 psychotic disorders, while controlling for gender and parental history of psychosis. Compared with no drug use, the use of cannabis only did not increase the risk of psychosis while the odds ratio for cannabis and other drug were statistically significant.
\end{abstract}

Research limitations/implications - Psychosis risk may be associated with the cumulative effect of polydrug use.

Practical implications - Cannabis use may be a proxy for other drug use in research studies.

Originality/value - This study is innovative as it uses linked self-report and administrative data for a large sample. Administrative data were used to as an objective mental health status indicator.

Keywords Logistic regression, Psychosis, Cannabis, Data linkage, Polydrug use, Registry data Paper type Research paper

\section{Introduction}

An association between cannabis use and psychosis has been well-documented in the epidemiological literature (Gage et al. , 2016). Indeed, a recent meta-analysis found evidence of a dose-response relationship, in which the heaviest users (in terms of both frequency and strength of substance consumed) were almost four times as likely to experience clinical-level psychotic outcomes compared with non-users (Marconi et al., 2016). Despite such findings, the potential causal role of cannabis use in the development of psychosis continues to be debated. There are a number of methodological challenges that impede the investigation of this association (Ksir and Hart, 2016). In the absence of experimental studies, it is difficult to isolate the unique effect (if any) that cannabis consumption has on psychosis (Ksir and Hart, 2016). With regards to epidemiological evidence, it is worth noting that while an association has consistently been reported, cannabis is the most the most commonly used recreational drug worldwide (Morley et al., 2015; National Institutes of Health, 2015; Haberstick et al., 2014; Degenhardt et al., 2013), and it is associated with many other well-documented risk factors for psychosis (Ksir and Hart, 2016) such as a pre-existing vulnerability to psychosis (Power et al., 2014). In their review of the extant literature, and paying attention to the Bradford Hill (1965) criteria for inferring causality from epidemiological data, Ksir and Hart (2016, p. 11) concluded that cannabis use in itself was not a definitive causal factor for psychosis, rather that "[... ] early use and heavy use of cannabis are more likely in individuals with a vulnerability to psychosis". 
One methodological challenge that has received relatively little interest in the literature is the issue of polydrug use. Cannabis use is the most commonly used drug within a polydrug context; studies of typologies of polydrug use have observed high levels of cannabis use across a range of groups that differ both qualitatively (in terms of the types of substances consumed) and quantitatively (in terms of their class counts, and associations with physical and mental health outcomes) (Stefanis et al., 2014; Smith et al., 2011; Fergusson et al., 2006; Lynskey et al., 2006; Carlson et al., 2005; Mitchell and Plunkett, 2000). While there have been many plausible hypotheses to explain the biological mechanisms that link cannabis use and psychosis (Solowij et al., 2013; Bhattacharyya et al., 2009; Rais et al., 2008; Szeszko et al., 2007; Linszen and van Amelsvoort, 2007; D'Souza et al., 2000) research suggests that psychosis is also related to the use of other illicit drugs. For example, stimulants (Sara et al., 2014; Medhus et al., 2015) and hallucinogens (Marona-Lewicka et al., 2011) have also been identified as risk factors for the development of psychotic disorders/experiences. As cannabis use frequently co-occurs with the use of other drugs that have been shown to be associated with psychosis risk, and given that cannabis is the most widely used recreational drug, it is possible that the unique deleterious effect of cannabis use may have been overstated in previous research.

Despite the fact that cannabis and other illicit substances are frequently used together, the association between cannabis use and psychosis has rarely been studied in a polydrug context. While a number of studies have found associations between cannabis use and psychosis, even after controlling for the presence of other drugs, the findings have been somewhat inconsistent and there were significant methodological differences between these studies. van Os et al. (2002) examined the association between self-reported cannabis use and any psychotic disorder in general population $(n=4,045)$ and clinical $(n=59)$ samples followed over a three year period. A strong, effect of cannabis use on later psychotic disorder was observed, and was not attenuated when other drug use was included in the model. Henquet et al. (2006) examined the relation between cannabis use and psychotic symptoms in individuals with above average predisposition for psychosis who first used cannabis during adolescence $(n=2,437)$. The inclusion of other drug use and predisposition to psychosis led to an attenuated effect, with the adjusted point estimate moderate in strength $(\mathrm{OR}=1.67)$. Arguably the most comprehensive study which controlled for other drug use was conducted by Zammit et al. (2002). Using Swedish conscript data $(n=50,087)$ they examined the association between self-reported cannabis and other drug use and later psychiatric admissions, assessed using data linkage. Overall, cannabis use had a dose-response association with psychotic diagnosis, even in those who reported using only cannabis, however the inclusion of other drugs led to attenuated effects. Gage et al. (2014) examined the association between cannabis use and subsequent psychotic experiences (PLEs) in a cohort of adolescents $(n=1,756)$. They found that, when the confounding effects of tobacco, alcohol, and other drugs were controlled for, the effect of cannabis use on PLE dropped to a relatively minor level $(O R=1.25)$.

The majority of studies that have examined the cannabis-psychosis association have treated other drug use as a covariate. Fewer studies have explicitly examined the impact of combining cannabis with other substances on the subsequent development of psychosis. Studies that have taken this approach have found that such a combination resulted in considerably higher levels of risk compared with cannabis use alone. For example, Van Dam et al. (2008) examined schizotypal symptoms in users of both legal and illicit substances $(n=328)$. Participants were divided into three groups based on their drug use patterns; legal drug only, cannabis and legal drug, cannabis polydrug. Those in the legal drug and cannabis-legal drug groups did not differ significantly on self-reported schizotypy, whereas those in the polydrug group scored significantly higher. Similarly, in a community cohort followed over 30 years $(n=591)$, Rössler et al. (2012) found considerably stronger associations between "multiple-drug use" (i.e. cannabis plus at least one other drug) and psychotic experiences, compared with the use of cannabis alone. This effect was particularly strong when the analyses focussed on "schizophrenia nuclear symptoms" suggesting that polydrug use may be a risk for more severe, clinical levels of psychosis. Given that these studies have focussed primarily on self-reported psychotic-like experiences, further research is required exploring the impact of polydrug use on clinical psychosis.

The main aim of this study was to assess the association between patterns of recreational drug use (no drug use, cannabis only, cannabis and other drug) and psychotic disorders. Data from a 
large interview survey based on a stratified random probability sample of young Danish people aged 24 years was used to assess life-time drug use and this information was linked to the Danish psychiatric registry for identification of participants who had a life-time ICD-10 diagnosis of a psychotic disorder and also to control for parental psychotic disorder. It was predicted that cannabis use and polydrug use would both be significantly associated with a diagnosis of a psychotic disorder while controlling for parental psychosis. It was expected that the effect size would be larger for polydrug use compared to cannabis only.

\section{Method \\ Participants and procedures}

Information on drug use was collected as part of a Danish national study conducted by The Danish National Centre for Social Research between 2008 and 2009. The aim of this study was to gather mental health-related data from young Danish people. A stratified random probability sample $(n=4,718)$ drawn from the entire birth cohort of Danes born in 1984 (participants aged 24 years) were contacted (participants aged 24 years and 2,980 individuals agreed to be interviewed. The response rate was 63 per cent. Participation in the interview was voluntary and the study was approved by the Danish Data Protection Agency. To increase the number of participants, who had been victims of childhood abuse and neglect, children who had been in child protection, were over-sampled by a ratio of 1:2 of "child protection cases" vs "non-child protection cases". A child protection case was defined as a case when the local authority (according to the files of local social workers) had provided support for the child and the family or placement with a foster family due to concerns about the well-being and development of the child. A total of 852 interviews were conducted with individuals who had been previously identified by the Danish authorities as child protection cases. To account for the oversampling of child protection cases and to ensure findings were representative of the total Danish population of young people aged 24 , the data were analysed using a weight variable with a weighted child protection status of $6.3 \%$ of the total sample. A structured interview was conducted by telephone, or by residential visit if telephone contact could not be made (mean duration of interview was 43 minutes). All individuals who volunteered to take part in the interview received written contact prior to the interview informing them of the process of the interview, the nature of the questions to be asked, and the process of confidentiality. All interviewers were formally trained and instructed by The Danish National Centre for Social Research and participated in test trials to become familiar with the questionnaire and the coding procedures.

The survey responses were linked to data from the Danish Civil Registration System and the Danish Psychiatric Central Register. A detailed description of the structure of CRS was provided by Pedersen et al. (2006). Access to the CRS was provided by Denmark Statbank upon completion of a research proposal. The relevant variables requested are matched to individuals using the person's civil registry number (CPR). The CPR identifies people at the individual level and allows information to be collated across different registries.

\section{Measures}

The survey included a section on drug use. Participants were asked "Have you ever tried[...]" and the following drugs were listed: amphetamine (Speed), cannabis (hashish/pot), cocaine (coke/ crack), LSD (acid), mushrooms with narcotic effect, heroin, solvents (sniffing), other (ecstasy). Each drug was scored "Yes" (1) or "No" (0). The scores were recoded into a categorical variable that represented: no drug use, cannabis only, and cannabis and any other drug.

The outcome variable was a diagnosis of psychotic disorder recorded between the years 1984 and 2005 (approximating an age range from birth to 21 years). Every time a person has contact with a psychiatric hospital or department in Denmark they receive an ICD-10 (previously ICD-8/9) diagnosis code that is recorded on the Psychiatric Central Register. The diagnosis is made by a psychiatrist. For this study we used information from the Psychiatric Central Register to identify which participants had received a diagnosis of any psychotic disorder (ICD-10 F20-F29; ICD8/9 295, 298.09, 298.19, 298.29, 298.39, 298.89, 297, 298.99, 299). These data are available as 
part of the Danish Civil Registration System (CRS) and the Danish Psychiatric Central Register. Parental data for all was also available from four years prior to birth (1980) to 2005; parental psychosis was indicated if either parent had a ICD-10 (F2O-F29) diagnosis during this time period.

\section{Analysis}

$\chi^{2}$ tests were used to examine the bivariate associations between the predictor and outcome variables. Binary logistic regression analysis was used to examine the association between drug use category and psychotic disorder. Analyses were conducted using SPSS version 21 (IBM Corp., 2012).

\section{Results}

Slightly more than half of the sample were male (52.2 per cent), 14 ( 0.5 per cent) had received a diagnosis of a psychotic disorder, and there were 45 ( 1.5 per cent) cases of parental diagnosis of psychosis. Almost half of the participants reported no drug use (48.4 per cent), a further 31.6 per cent reported using cannabis only and 20.0 per cent reported using cannabis and any other drug. The bivariate associations between the psychosis diagnosis and other variables are reported in Table I.

Table I shows that there was no association between psychosis diagnosis and gender or parental psychosis. There was a significant association between psychosis diagnosis and drug use with more participants with a diagnosis having reported using cannabis and other drug(s). The variables were entered into a binary logistic regression with the psychosis diagnosis as the dependent variable. Gender, parental psychosis and drug use were entered as predictors with the "No drug" category used as the reference level. The model was statistically significant $\left(\chi^{2}=13.68\right.$, df $=4, p<0.05$; Cox and Snell $R^{2}=0.004$; Nagelkerke $R^{2}=0.07$ ). The results in Table II show that, compared to the no drug use group, cannabis use only did not increase the risk of psychosis while the odds ratio for was cannabis and other drug were statistically significant ( $\mathrm{OR}=5.96)$.

In order to determine which drugs in combination with cannabis were contributing to this effect a series of $\chi^{2}$ analyses were conducted. The drug use data were recoded into seven binary variables to represent the use of: amphetamine with cannabis; cocaine with cannabis; LSD with cannabis; mushrooms with cannabis; heroin with cannabis; solvents with cannabis; and other

Table I Cross-tabulation of psychosis diagnosis, gender, parental psychosis and drug use

\begin{tabular}{lcccc} 
& \multicolumn{4}{c}{ Psychosis diagnosis count (\%) } \\
& No $(n=2,964)$ & Yes $(n=14)$ & $\chi^{2}(d f)$ & $p$ \\
\hline Gender (Male) & $1,546(52.2 \%)$ & $7(50.0 \%)$ & $0.03(1)$ & 0.87 \\
Parental psychosis & $45(1.5 \%)$ & $0(0 \%)$ & $0.22(1)$ & 0.64 \\
Drug use & & & & \\
No drug use & $1,437(48.5 \%)$ & $4(28.6 \%)$ & & \\
Cannabis only & $940(31.7 \%)$ & $2(14.3 \%)$ & & \\
Cannabis and other drug & $586(19.8 \%)$ & $8(57.1 \%)$ & $12.23(2)$ & $p<0.01$
\end{tabular}

\begin{tabular}{|c|c|c|c|c|}
\hline & $B$ & Sig. & $O R$ & $95 \% \mathrm{Cl}$ \\
\hline Gender (Male) & -0.56 & 0.32 & 0.57 & $(0.19-1.72)$ \\
\hline Parental Psychosis & 0.64 & 0.68 & 1.90 & (0.09-39.81) \\
\hline \multicolumn{5}{|c|}{ No drug use (reference category) } \\
\hline Cannabis only & -0.37 & 0.68 & 0.69 & $(0.12-4.07)$ \\
\hline Cannabis and other drug & 1.79 & 0.01 & 5.96 & $(1.71-20.75)$ \\
\hline
\end{tabular}


drug with cannabis. $\chi^{2}$ tests were used to test the association between these variables and psychosis diagnosis. The chi-square tests were significant for amphetamine with cannabis $\left(\chi^{2}=6.30, \mathrm{df}=1, p<0.05 ; \mathrm{OR}=3.7195 \% \mathrm{Cl} 1.24-11.14\right)$, cocaine with cannabis $\left(\chi^{2}=12.96\right.$, $\mathrm{df}=1, p<0.01 ; \mathrm{OR}=5.6695 \% \mathrm{Cl} 1.95-16.42)$, mushrooms with cannabis $\left(\chi^{2}=20.00, \mathrm{df}=1\right.$, $p<0.01 ; \mathrm{OR}=8.2695 \% \mathrm{Cl} 2.74-24.89)$, and solvents with cannabis $\left(\chi^{2}=10.57, \mathrm{df}=1\right.$, $p<0.01 ; \mathrm{OR}=5.1595 \% \mathrm{Cl} 1.71-15.46)$. Finally, to examine the effect of the number of different drugs used a variable that was computed that represented the total number of different drugs used, ranging from 0 (no drug use) to 7 (all drugs). This variable was entered as a predictor in a binary logistic regression with psychosis as the dependent variable. The model was statistically significant $\left(\chi^{2}=7.20, \mathrm{df}=1, p<0.01\right)$ and the odds ratio for the number of different drugs used was $1.38(95 \% \mathrm{Cl} 1.11-1.68)$. This indicates that each additional drug that is used increases the likelihood of a diagnosis of psychosis.

\section{Discussion}

The main aim of this study was to assess the association between patterns of recreational drug use (no drug use, cannabis only, cannabis and other drug) and psychotic disorders. It was predicted that cannabis use alone and polydrug use would both be significantly associated with a diagnosis of a psychotic disorder while controlling for parental psychosis. It was also expected that the effect size would be larger for polydrug use compared to cannabis only. The results partially supported the hypotheses. Although there was a significant bivariate association between psychosis diagnosis and drug use, with more participants with a diagnosis having reported using cannabis and other drugs, the logistic regression showed that there was no significant effect for cannabis only. Only polydrug use was significantly related to psychosis, with the odds increasing by almost six times compared to the "no drug" group. Follow-up analyses showed that different combinations of cannabis and other drugs were associated with psychosis; cannabis in combination with amphetamine, cocaine, mushrooms and solvents were all significantly associated with psychosis. There was also a significant dose-response relationship for the number of different drugs used and psychosis.

Although a large number of previous studies have examined the relationship between cannabis use and subsequent psychosis, the use of other drugs has largely been reduced to covariate status (e.g. Gage et al., 2014; Henquet et al., 2006; van Os et al., 2002; Zammit et al., 2002). Studies that have controlled for the presence of other drugs have delivered equivocal results, with some reporting moderate-to-strong associations between cannabis use and subsequent psychosis (van Os et al., 2002; Zammit et al., 2002; Henquet et al., 2006), while others have reported greatly attenuated effects (Gage et al., 2014). Studies that have tested specific permutations of polydrug use have suggested that the combination of cannabis with other substances leads to markedly increased risk for psychosis. Van Dam et al. (2008), found those who used legal drugs alone and those who combined legal drugs with cannabis did not differ significantly on a self-report measure of schizotypy, while those who combined cannabis with other illegal drugs scored significantly higher. Similarly, Rössler et al. (2012) examined data from a Swedish cohort followed over a thirty year period and found that polydrug users had a significantly greater risk of developing psychotic experiences compared with those who used cannabis alone. Both of these studies used sub-clinical measures of psychosis. The present study adds to the literature by demonstrating a similar association when a clinical diagnosis of psychotic disorder is used as the primary outcome measure. This provides indirect evidence for the "psychosis continuum" (Strauss, 1969; van Os et al., 2000) as it suggests that risk factors for psychosis operate in a consistent manner at both clinical and sub-clinical levels of psychosis.

The findings of the present study indicate that, when patterns of cannabis and polydrug use are examined in greater detail, the unique effect of cannabis consumption is greatly attenuated. As such, the effect of cannabis use on the development of psychosis may have been overstated in previous studies which failed to control for confounding effects of other drugs. One explanation for the consistent findings of an association between cannabis and psychosis is that cannabis use is a proxy for other drug use, and it may be that other drugs represent the true risk factor. Indeed, in the present study the number of different drugs used was associated with psychosis in a "dose-response" fashion. However, there does not appear to be specificity as different drugs 
have been shown to be associated with increased risk of psychosis or psychotic-like experiences; methamphetamine (McKetin et al., 2010), cocaine (Thirthalli and Benegal, 2006), and psychedelics (Kuzenko et al., 2011). The present findings highlight the importance of considering cannabis use within a broader polydrug context when attempting to infer causal links with psychosis. There are also clinical implications associated with these findings. It would be preemptive to state that smoking cannabis is a benign activity in relation to mental health, as much more research is required. In addition, the negative social, cognitive and physical consequences of cannabis use have been well documented (see review by Hall, 2015).

Although cannabis use may be a proxy indicator for other forms of drug use, it is also likely to be associated (or interact) with other risk factors for psychosis such as childhood trauma (Houston et al., 2011), familial risk for psychosis (Giordano et al., 2014), ethnicity and social disadvantage (Morgan et al., 2009). Therefore, future studies of the effects of cannabis should place cannabis use within a broader context of correlated risk-factors, such as the social defeat model (Selten et al., 2013).

Although it may be difficult to isolate the unique effect of cannabis use on psychosis using epidemiological methods, alternative methods may be of use. Up until very recently, experimental studies using human subjects were all but impossible given the legal status of cannabis. Although animal studies offer some insight, there is no reliable model of psychosis in animals, making the generalisation of findings difficult (Murray and DiForti, 2016). Following the legalisation of recreational marijuana in various parts of the USA in 2016, there will be increasing opportunities to test for causal associations between cannabis use and psychosis. This presents an opportunity to test different aspects of cannabis use that have been implicated in the "dose-response" relationship, e.g. frequency, type and strength (Marconi et al., 2016).

The findings of the present study should be considered in light of the following limitations. First, drug use was assessed using retrospective self-reports, which are open to under-or over-reporting. Second, the temporal ordering of drug use and diagnosis of psychosis cannot be established. Third, participant information about the frequency, type or strength of cannabis use was not available, meaning the dose-response nature of the cannabis-psychosis association could not be examined. Fourth, information regarding psychotic diagnosis was only available up until age 21, yet it is relatively common for psychosis to emerge up to the age of 35 years (Kessler et al., 2007). As such, the lifetime prevalence rate for psychotic disorder was relatively low in the present sample. Further research over a greater age range is recommended. Fifth, the genetic risk for psychosis was only approximated by using data for parental diagnosis of psychosis. Data were limited to a recorded diagnosis anytime from four years prior to the birth of the study child until the child was assessed aged 21, likely leading to an underestimation of parental psychosis. Finally, the cell counts for the psychosis variable are very unbalanced, with a small number of psychosis cases. This can cause problems with the estimation of the logistic model. The effects of "rare event" outcomes have been shown to produce bias in the estimates by underestimating the probability of the outcome variable (King and Zeng, 2001). The non-significant effect for the "Cannabis only" category of the drug use variable should be interpreted in light of the potential of a Type 2 error occurring.

In summary, this study aimed to assess the relationship between patterns of recreational drug use (no drug use, cannabis only, cannabis and other drug) and psychotic disorders. There was no significant effect for cannabis alone, but cannabis in conjunction with other drugs was statistically significant. Follow-up analyses indicated that polydrug use was significantly associated with increased risk of psychosis. Future research should address the context of cannabis use, both as part of more complex patterns of drug use but also in the context of a broader set of social, economic, and psychological risk factors.

\section{References}

Bhattacharyya, S., Fusar-Poli, P., Borgwardt, S., Martin-Santos, R., Nosarti, C., O'Carroll, C., et al. (2009), "Modulation of mediotemporal and ventrostriatal function in humans by $\Delta 9$-tetrahydrocannabinol", Archives of General Psychiatry, Vol. 66 No. 4, pp. 442-51, doi: 10.1001/archgenpsychiatry.2009.17.

Carlson, R., Wang, J., Falck, R. and Siegal, H. (2005), "Drug use practices among MDMA/ecstasy users in Ohio: a latent class analysis", Drug and Alcohol Dependence, Vol. 79 No. 2, pp. 167-79, doi: 10.1016/j. drugalcdep.2005.01.011. 
D’Souza, D., Abi-Saab, W., Madonick, S., Wray, Y., Forselius, K., MacDougall, L., et al. (2000), "Cannabinoids and psychosis: evidence from studies with I.V THC in schizophrenic patients and controls", Schizophrenia Research, Vol. 41 No. 1, 1pp., doi: 10.1016/s0920-9964(00)90371-2.

Degenhardt, L., Ferrari, A.J., Calabria, B., Hall, W.D., Norman, R.E., McGrath, J., Flaxman, A.D., Engell, R.E., Freedman, G.D., Whiteford, H.A. and Vos, T. (2013), "The global epidemiology and contribution of cannabis use and dependence to the global burden of disease: results from the GBD 2010 study", Plos ONE, Vol. 8 No. 10, p. e76635, available at: https://doi.org/10.1371/journal.pone.0076635

Fergusson, D., Boden, J. and Horwood, L. (2006), "Cannabis use and other illicit drug use: testing the cannabis gateway hypothesis", Addiction, Vol. 101 No. 4, pp. 556-69, doi: 10.1111/j.13600443.2005.01322.x.

Gage, S., Hickman, M. and Zammit, S. (2016), "Association between cannabis and psychosis: epidemiologic evidence”, Biological Psychiatry, Vol. 79 No. 7, pp. 549-56, doi: 10.1016/j.biopsych.2015.08.001.

Gage, S., Hickman, M., Heron, J., Munafò, M., Lewis, G., Macleod, J. and Zammit, S. (2014), “Associations of cannabis and cigarette use with psychotic experiences at age 18: findings from the Avon longitudinal study of parents and children", Psychological Medicine, Vol. 44 No. 16, pp. 3435-44, doi: 10.1017/ s0033291714000531.

Giordano, G.N., Ohlsson, H., Kendler, K.S., Sundquist, K. and Sundquist, J. (2014), "Unexpected adverse childhood experiences and subsequent drug use disorder: a Swedish population study (1995-2011)", Addiction, Vol. 109 No. 7 pp. 1119-27, doi: org/10.1371/journal.pone.0151755.

Haberstick, B., Young, S., Zeiger, J., Lessem, J., Hewitt, J. and Hopfer, C. (2014), "Prevalence and correlates of alcohol and cannabis use disorders in the United States: results from the national longitudinal study of adolescent health", Drug and Alcohol Dependence, Vol. 136, pp. 158-61, doi: 10.1016/j. drugalcdep.2013.11.022.

Hall, W. (2015), "What has research over the past two decades revealed about the adverse health effects of recreational cannabis use?", Addiction, Vol. 110 No. 1, pp. 19-35, doi: 10.1111/add.12703.

Henquet, C., Krabbendam, L., Spauwen, J., Kaplan, C., Lieb, R., Wittchen, H. and Van Os, J. (2006), "Prospective cohort study of cannabis use, predisposition for psychosis, and psychotic symptoms in young people", European Neuropsychopharmacology, Vol. 16, p. S381, doi: 10.1016/s0924-977x(06)70460-6.

Hill, A. (1965), "The environment and disease: association or causation?", Proceedings of the Royal Society of Medicine, Vol. 58 No. 5, pp. 295-300.

Houston, J.E., Murphy, J., Shevlin, M. and Adamson, G. (2011), "Cannabis use and psychosis: re-visiting the role of childhood trauma", Psychological Medicine, Vol. 41 No. 11, pp. 2339-48, doi: 10.1017/S0033291711000559.

IBM Corp (2012), IBM SPSS Statistics for Windows, Version 21.0, IBM Corp, Armonk, NY.

Kessler, R., Amminger, G., Aguilar-Gaxiola, S., Alonso, J., Lee, S. and Ustun, T. (2007), "Age of onset of mental disorders: a review of recent literature", Current Opinion in Psychiatry, Vol. 20 No. 4, pp. 359-64, doi: 10.1097/yco.0b013e32816ebc8c.

King, G. and Zeng, L. (2001), "Logistic regression in rare events data”, Political Analysis, Vol. 9 No. 2, pp. 137-63.

Ksir, C. and Hart, C. (2016), "Cannabis and psychosis: a critical overview of the relationship", Current Psychiatry Reports, Vol. 18 No. 2, doi: 10.1007/s11920-015-0657-y.

Kuzenko, N., Sareen, J., Beesdo-Baum, K., Perkonigg, A., Höfler, M., Simm, J., Lieb, R. and Wittchen, H.-U. (2011), "Associations between use of cocaine, amphetamines, or psychedelics and psychotic symptoms in a community sample", Acta Psychiatrica Scandinavica, Vol. 123 No. 6, pp. 466-74, doi: 10.1111/j.16000447.2010.01633.x.

Linszen, D. and van Amelsvoort, T. (2007), "Cannabis and psychosis: an update on course and biological plausible mechanisms", Current Opinion in Psychiatry, Vol. 20 No. 2, pp. 116-20, doi: 10.1097/yco.0b013e32803577fb.

Lynskey, M., Agrawal, A., Bucholz, K., Nelson, E., Madden, P., Todorov, A., et al. (2006), "Subtypes of illicit drug users: a latent class analysis of data from an Australian twin sample", Twin Research and Human Genetics, Vol. 9 No. 04, pp. 523-30, doi: 10.1375/twin.9.4.523.

McKetin, R., Hickey, K., Devlin, K. and Lawrence, K. (2010), "The risk of psychotic symptoms associated with recreational methamphetamine use”, Drug and Alcohol Review, Vol. 29 No. 4, pp. 358-63, doi: 10.1111/ j.1465-3362.2009.00160.x. 
Marconi, A., Di Forti, M., Lewis, C., Murray, R. and Vassos, E. (2016), "Meta-analysis of the association between the level of cannabis use and risk of psychosis", Schizophrenia Bulletin, Vol. 42 No. 5, pp. 1262-9, doi: 10.1093/schbul/sbw003.

Marona-Lewicka, D., Nichols, C. and Nichols, D. (2011), "An animal model of schizophrenia based on chronic LSD administration: old idea, new results”, Neuropharmacology, Vol. 61 No. 3, pp. 503-12, doi: 10.1016/j. neuropharm.2011.02.006.

Medhus, S., Rognli, E., Gossop, M., Holm, B., Mørland, J. and Bramness, J. (2015), "Amphetamine-induced psychosis: transition to schizophrenia and mortality in a small prospective sample", The American Journal on Addictions, Vol. 24 No. 7, pp. 586-9, doi: 10.1111/ajad.12274.

Mitchell, C. and Plunkett, M. (2000), "The latent structure of substance use among American Indian adolescents: an example using categorical variables", American Journal of Community Psychology, Vol. 28 No. 1, pp. 105-25, doi: 10.1023/a:1005146530634.

Morgan, C., Fisher, H., Hutchinson, G., Kirkbride, J., Craig, T.K., Morgan, K., Dazzan, P., Boydell, J., Doody, G.A., Jones, P.B., Murray, R.M., Leff, J. and Fearon, P. (2009), "Ethnicity, social disadvantage and psychotic-like experiences in a healthy population based sample", Acta Psychiatrica Scandinavica, Vol. 119 No. 3, pp. 226-35, doi: 10.1111/j.1600-0447.2008.01301.x.

Morley, K.I., Lynskey, M.T., Moran, P., Borschmann, R. and Winstock, A.R. (2015), "Polysubstance use, mental health and high-risk behaviours: results from the 2012 Global Drug Survey", Drug and Alcohol Review, Vol. 34 No. 4, pp. 427-37, doi: 10.1111/dar.12263.

Murray, R.M. and Di Forti, M. (2016), "Cannabis and psychosis: what degree of proof do we require?", Biological Psychiatry, Vol. 79 No. 7, pp. 514-5, doi: 110.1016/j.biopsych.2016.02.005.

National Institutes of Health (2015, October), NIH News Releases, National Institutes of Health website, available at: www.nih.gov/news-events/news-releases/prevalence-marijuana-use-among-us-adultsdoubles-over-past-decade

Pedersen, C.B., Gotzsche, H., Moller, J.O. and Mortensen, P.B. (2006), "The Danish Civil Registration System. a cohort of eight million persons", Danish Medical Bulletin, Vol. 53 No. 4, pp. 441-9.

Power, R.A., Verweij, K.J., Zuhair, M., Montgomery, G.W., Henders, A.K., Heath, A.C., Martin, N.G., Madden, P.A., Medland, S.E. and Wray, N.R. (2014), "Genetic predisposition to schizophrenia associated with increased use of cannabis”, Molecular Psychiatry, Vol. 19 No. 11, pp. 1201-4, doi: 10.1038/mp.2014.51.

Rais, M., Cahn, W., Van Haren, N., Schnack, H., Caspers, E., Hulshoff Pol, H. and Kahn, R. (2008), "Excessive brain volume loss over time in cannabis-using first-episode schizophrenia patients", American Journal of Psychiatry, Vol. 165 No. 4, pp. 490-6, doi: 10.1176/appi.ajp.2007.07071110.

Rössler, W., Hengartner, M., Angst, J. and Ajdacic-Gross, V. (2012), "Linking substance use with symptoms of subclinical psychosis in a community cohort over 30 years", Addiction, Vol. 107 No. 6, pp. 1174-84, doi: 10.1111/j.1360-0443.2011.03760.x.

Sara, G., Burgess, P., Malhi, G., Whiteford, H. and Hall, W. (2014), "Stimulant and other substance use disorders in schizophrenia: prevalence, correlates and impacts in a population sample", Australian \& New Zealand Journal of Psychiatry, Vol. 48 No. 11, pp. 1036-47, doi: 10.1177/0004867414533838.

Selten, J.P., van der Ven, E., Rutten, B.P. and Cantor-Graae, E. (2013), "The social defeat hypothesis of schizophrenia: an update", Schizophrenia Bulletin, Vol. 39 No. 6, pp. 1180-6.

Smith, G., Farrell, M., Bunting, B., Houston, J. and Shevlin, M. (2011), "Patterns of polydrug use in great Britain: findings from a national household population survey", Drug and Alcohol Dependence, Vol. 113 Nos 2/3, pp. 222-8, doi: 10.1016/j.drugalcdep.2010.08.010.

Solowij, N., Walterfang, M., Lubman, D., Whittle, S., Lorenzetti, V., Styner, M., Velakoulis, D., Pantelis, C. and Yücel, M. (2013), "Alteration to hippocampal shape in cannabis users with and without schizophrenia", Schizophrenia Research, Vol. 143 No. 1, pp. 179-84, doi: 10.1016/j.schres.2012.10.040.

Stefanis, N., Dragovic, M., Power, B., Jablensky, A., Castle, D. and Morgan, V. (2014), "The effect of drug use on the age at onset of psychotic disorders in an Australian cohort", Schizophrenia Research, Vol. 156 Nos 2/3, pp. 211-6, available at: http://dx.doi.org/10.1016/j.schres.2014.04.003

Strauss, J.S. (1969), "Hallucinations and delusions as points on continua function", Archives of General Psychiatry, Vol. 21 No. 5, pp. 581-6. 
Szeszko, P., Robinson, D., Sevy, S., Kumra, S., Rupp, C., Betensky, J., Lencz, T., Ashtari, M., Kane, J.M., Malhotra, A.K., Gunduz-Bruce, H., Napolitano, B. and Bilder, R.M. (2007), "Anterior cingulate grey-matter deficits and cannabis use in first-episode schizophrenia", The British Journal of Psychiatry, Vol. 190 No. 3, pp. 230-36, doi: 10.1192/bjp.bp.106.024521.

Thirthalli, J. and Benegal, V. (2006), "Psychosis among substance users", Current Opinion in Psychiatry, Vol. 19 No. 3, pp. 239-45, doi: 10.1097/01.yco.0000218593.08313.fd.

Van Dam, N., Earleywine, M. and DiGiacomo, G. (2008), "Polydrug use, cannabis, and psychosis-like symptoms", Human Psychopharmacology: Clinical and Experimental, Vol. 23 No. 6, pp. 475-85, doi: 10.1002/hup.950.

van Os, J., Bak, M., Hanssen, M., Bijl, R., de Graaf, R. and Verdoux, H. (2002), "Cannabis use and psychosis: a longitudinal population-based study", American Journal of Epidemiology, Vol. 156 No. 4, pp. 319-27, doi: 10.1093/aje/kwf043.

van Os, J., Hanssen, M., Bijl, R.V. and Ravelli, A. (2000), "Straus (1969) revisited: a psychosis continuum in the general population?", Schizophrenia Research, Vol. 45 Nos 1-2, pp. 11-20, doi: 10.1017/ S0033291708003814.

Zammit, S., Allebeck, P., Andreasson, S., Lundberg, I. and Lewis, G. (2002), "Self reported cannabis use as a risk factor for schizophrenia in Swedish conscripts of 1969: historical cohort study”, BMJ, Vol. 325 No. 7374 , pp. 1199-204, doi: 10.1136/bmj.325.7374.1199.

\section{Further reading}

Grant, B., Saha, T., Ruan, W., Goldstein, R., Chou, S., Jung, J., et al. (2016), "Epidemiology of DSM-5 drug use disorder", JAMA Psychiatry, Vol. 73 No. 1, pp. 39-47, doi: 10.1001/jamapsychiatry.2015.2132.

\section{Corresponding author}

Mark Shevlin can be contacted at: m.shevlin@ulster.ac.uk

For instructions on how to order reprints of this article, please visit our website: 
Reproduced with permission of copyright owner. Further reproduction prohibited without permission. 